\title{
End Organ Damage from Diabetes
}

National Cancer Institute

\section{Source}

National Cancer Institute. End Organ Damage from Diabetes. NCI Thesaurus. Code C113717.

Irreparable damage to multiple organs/systems which is the result of chronic complications of diabetes. The affected organs/systems include the cardiovascular system, kidneys, eyes, nervous system, joints and feet. 